\section{Taquicardiomiopatía, causa reversible de insuficiencia cardiaca: caso clínico}

\author{
LITAL MEYER ${ }^{1}$, ROBERTO CONCEPCIÓN ${ }^{2}$, NIBALDO ZAMORANO ${ }^{2}$, \\ MACARENA ARMSTRONG ${ }^{1}$, LEONARDO CASTRO ${ }^{3, a}$, \\ MAXIMILIANO MUJICA ${ }^{3, a}$, RENE ASENJO $^{4}$
}

\section{Tachycardiomyopathy as a reversible cause of heart failure. Report of one case}

Tachycardiomyopathy is a potentially reversible cause of heart failure. It can be induced by supraventricular or ventricular arrhythmias. When these are treated, systolic function improves or normalizes. We report a 20 year-old male with deterioration of left ventricular function and dilated cardiomyopathy secondary to an incessant atrial tachycardia that was treated with radiofrequency catheter ablation. After the procedure, the patient experienced a significant improvement of his ventricular function.

(Rev Med Chile 2012; 140: 231-235).

Key words: Cardiomyopathies; Catheter ablation, radiofrequency; Tachycardia, supraventricular.

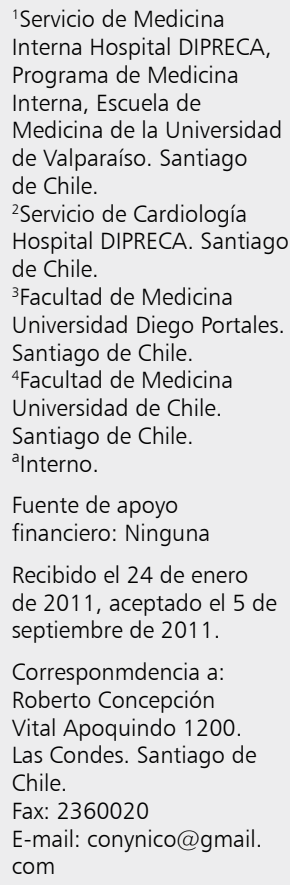

donde se le solicita radiografía de tórax, destacando gran cardiomegalia y signos de congestión pulmonar (Figura 1). Se contaba con examen físico y radiografía de tórax de 3 años atrás sin ninguna alteración.

Fue derivado a centro de atención terciaria al cuarto día de evolución del cuadro. Hemodinamia siempre estable. Se objetivó en electrocardiograma (ECG) taquicardia auricular intermitente (Figura $2)$, tratada inicialmente con amiodarona.

Ingresó a nuestro servicio hipotenso $(84 / 56$ $\mathrm{mmHg}$ ), taquicárdico (116 latidos por minuto, irregular), afebril, frecuencia respiratoria de 18 por minuto y saturando $92 \%$ con oxígeno ambiental, bien perfundido. La faringe se encontraba algo enrojecida, yugulares ingurgitadas. Sin congestión pulmonar y examen cardíaco demostró tercer ruido y ritmo de galope. Extremidades sin edema.

De los exámenes de laboratorio destacaba discreta leucocitosis, PCR baja, enzimas cardíacas $\mathrm{y}$ troponinas normales.

El ecocardiograma realizado en nuestra unidad mostró ventrículo izquierdo (VI) severamente dilatado $(82 / 74 \mathrm{~mm})$ con disfunción sistólica global severa [fracción de eyección (FE): 20\%], 
hipokinesia difusa, disfunción diastólica tipo III de IV. AI de $49 \mathrm{~mm}$, con disfunción sistólica ventricular derecha, leve dilatación de cavidades derechas e hipertensión pulmonar (HTP) moderada (Figura 3).

Se realizó resonancia magnética cardiaca que concluyó miocardiopatía dilatada, sin elementos de fibrosis ni necrosis, con disfunción sistólica severa.

Paciente se mantuvo taquicárdico, presión arterial en límite bajo, en tratamiento con inhibi-

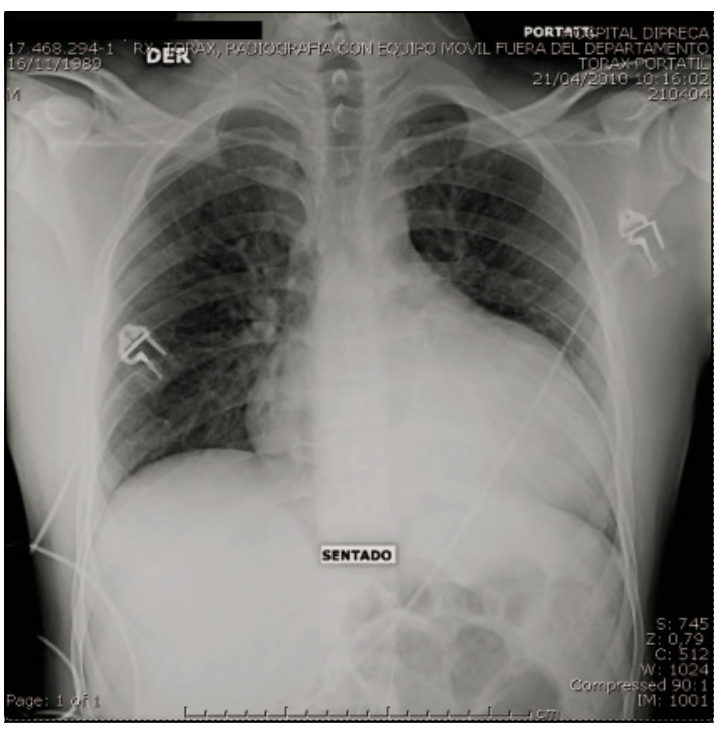

Figura 1. Radiografía de tórax al ingreso mostrando importante cardiomegalia. dores de la enzima convertidora de angiotensina (IECA) y betabloqueo en dosis bajas.

Dentro del estudio solicitado: función tiroidea normal; VHB (-), VHC (-), VIH (-), citomegalovirus (-), Chagas (-), IgM Mycoplasma pneumoniae $(+)$ Se trato con azitromicina por 5 días.

Se realizó biopsia endomiocárdica que concluyó cambios inespecíficos, compatibles con miocardiopatía dilatada, sin evidencias de enfermedad inflamatoria activa.

El Holter de ECG evidenció taquicardia auricular incesante sobre $90 \%$ del tiempo. Se planteo el diagnostico de taquicardiomiopatía. El tratamiento con amiodarona, bisoprolol y digitálicos no fueron efectivas en el manejo de la taquiarritmia. Se decidió mapeo y ablación de taquicardia auricular incesante usando el sistema EnSite $\mathrm{Navx}^{\mathrm{TM}}$. Se identificó y realizó ablación a foco asociado a vena cava superior y vena pulmonar superior derecha, convirtiendo a ritmo sinusal con 85 latidos por minuto (Figura 5).

Dado de alta a los 21 días de su admisión.

En los controles posteriores se ha mantenido en capacidad funcional I, ritmo sinusal, con frecuencia cardíaca alrededor de 70 latidos por minuto, regular y normotenso. A los 3 meses posterior a la ablación, el ecocardiograma evidenció marcada reducción de las cámaras cardiacas con mejoría de la función sistólica del VI y mínimo reflujo mitral. A los 6 meses el paciente se encuentra en capacidad funcional I y el ecocardiograma en límite normal con radiografía de tórax normal (Figuras 4 y 6).

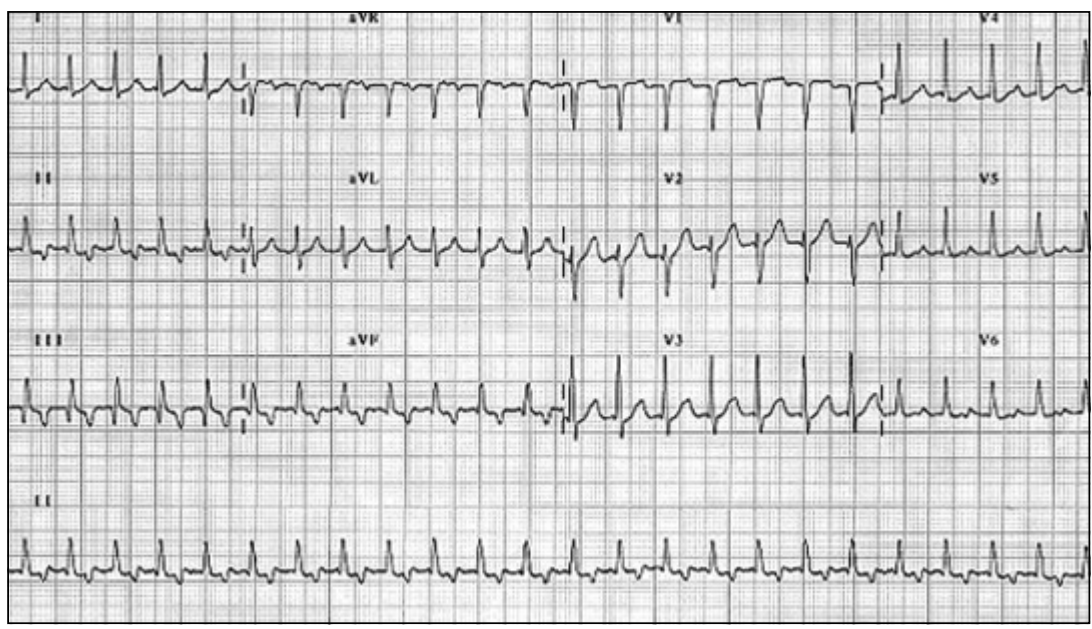

Figura 2. ECG al ingreso mostrando taquicardia auricular rápida. 


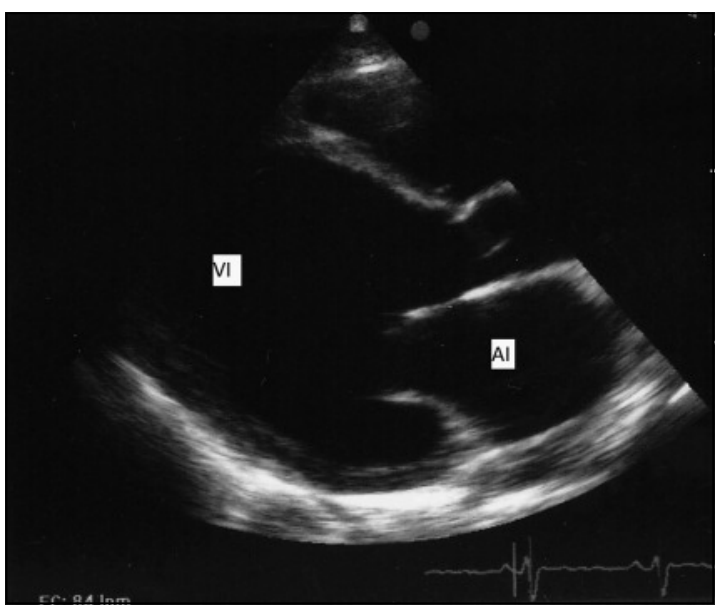

Figura 3. Ecocardiograma al ingreso. Eje largo paraesternal. Dilatación severa del VI (diámetro telediastólico de VI: $82 \mathrm{~mm}$ ). Al: aurícula izquierda. VI: ventrículo izquierdo.

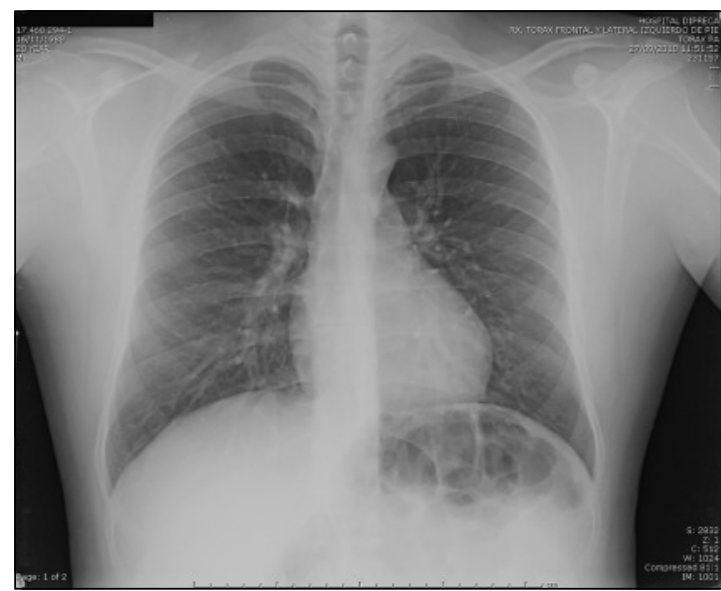

Figura 4. Radiografía de tórax a los 6 meses del cese de la arritmia, con regresión de la cardiomegalia.

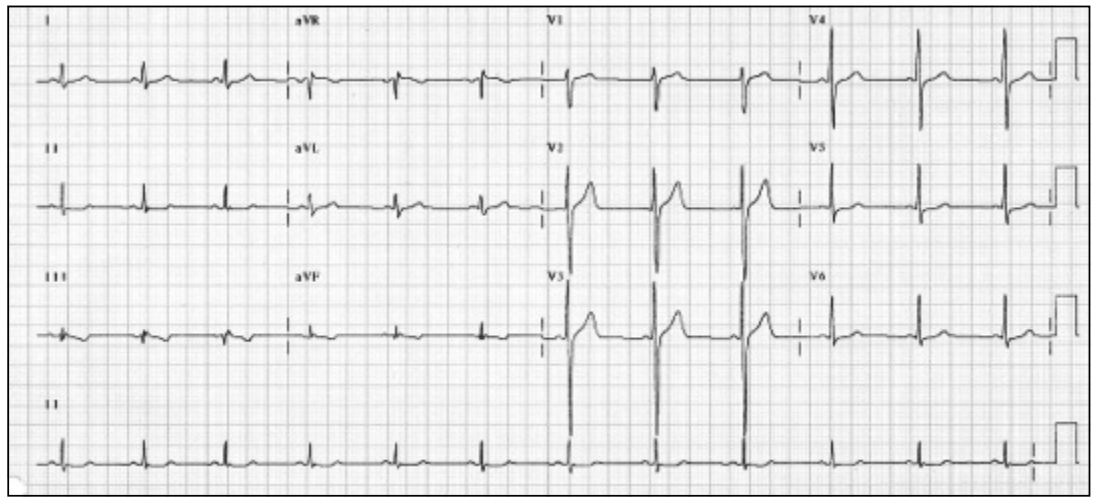

Figura 5. ECG posterior a la ablación del foco arritmogénico mostrando ritmo regular sinusal.

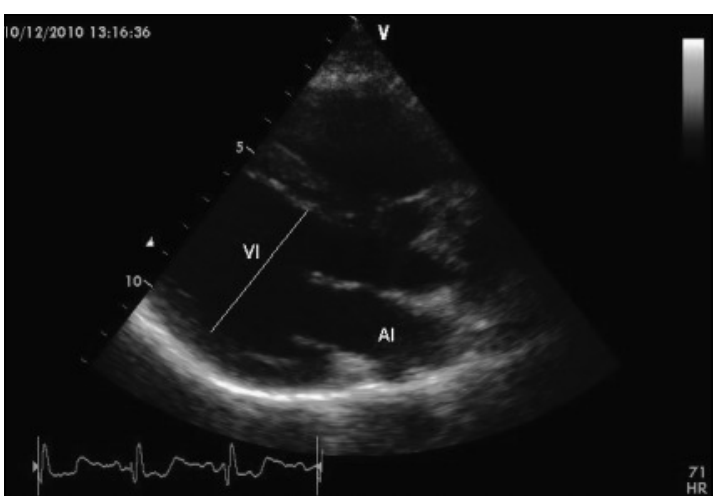

Figura 6. Ecocardiograma a los 6 meses posterior a la terapia ablativa. Eje largo paraesternal. VI de diámetro normal. Al: aurícula izquierda. VI: ventrículo izquierdo.

\section{Discusión}

La relación entre taquiarritmia y disfunción ventricular izquierda fue descrita por primera vez en 1949 por Phillips y Levine en pacientes con fibrilación auricular y falla cardiaca reversible ${ }^{4}$. La taquicardiomiopatía puede ser inducida por arritmias supraventriculares y ventriculares. Puede ser difícil definir la relación causa efecto cuando la miocardiopatía y la taquicardia son encontradas simultáneamente ${ }^{1-7}$.

La taquicardiomiopatía se puede clasificar en dos categorías: "puras" cuando la taquicardia crónica se instala en un corazón normal y es el único agente responsable de la disfunción del VI. 
Cuando esta condición no se cumple, la taquicardiomiopatía es "impura". En ambas formas la recuperación de la función ventricular es variable, desde completa hasta completamente ausente ${ }^{2}$.

La taquicardiomiopatía se puede presentar a cualquier edad y no tiene preferencia en relacion al genero. En pacientes con corazón normal, la taquiarritmia crónica puede ser muy bien tolerada y asintomática, solicitando asistencia médica recién cuando entran en falla cardíaca. Al contrario, los pacientes con cardiopatía de base reciben atención médica antes ya que estos son mucho más sintomáticos ${ }^{2,3}$.

No existen métodos específicos para identificar la presencia de una taquicardiomiopatía y el diagnóstico puede ser difícil de realizar. El diagnóstico correcto se puede establecer con certeza posterior a la normalización o mejoría de la disfunción ventricular izquierda con el cese o control de la taquiarritmia ${ }^{1,2,4}$. Sin embargo, es un hecho que el control de la taquiarritmia no siempre mejora la función ventricular en pacientes con taquicardiomiopatía y puede reflejar el estadio irreversible de esta patología, complicando así el diagnóstico.

La mejor herramienta para el diagnóstico es el alto índice de sospecha clínica en base a la historia y a los hallazgos clínicos ${ }^{1,2,4-7}$. Fenelon et $\mathrm{al}^{2}$ propusieron criterios para el diagnostico de taquicardiomiopatía: pacientes con 1) miocardiopatía dilatada o falla cardiaca; 2) arritmia cardiaca crónica o frecuente, incluyendo la taquicardia incesante supraventricular, fibrilación auricular o flutter y taquicardia ventricular incesante. En general una taquicardia que ocurre sobre un 10-15\% del día puede resultar en una miocardiopatía. La frecuencia ventricular que origina la taquicardiomiopatía no se ha determinado, aunque cualquier frecuencia cardiaca mayor a 100 latidos por minuto puede ser importante ${ }^{4}$.

En pacientes con taquicardiomiopatía se observan alteraciones hemodinámicos que incluyen disminución de la $\mathrm{FE}$, aumento de los volúmenes de fin de diástole y fin de sístole y aumento en las presiones de fin de diástole y de arteria pulmonar. Hay una activación neurohormonal intensa caracterizada por elevación marcada de los niveles de péptido natriurético auricular, elevadas concentraciones de epinefrina y norepinefrina, aumento de la actividad plasmática de renina y elevados niveles de aldosterona plasmática. El término de la taquicardia lleva a mejoría del cuadro clínico, de aumento en la FE y una marcada disminución de los volúmenes de fin de diástole ${ }^{1,4,7}$.

En relación al tratamiento de la taquicardiomiopatía, la piedra angular de ésta es el control de la taquiarritmia, ya que con esto puede mejorar la función ventricular izquierda y la falla cardia$\mathrm{ca}^{1,2,4,6,7}$. Este objetivo se puede lograr ya sea con tratamiento farmacológico o no farmacológico.

El fármaco de elección va a depender de la arritmia de base, con diversos resultados. Bohora et $\mathrm{al}^{8}$ describieron el caso de una paciente de 15 años con taquicardiomiopatía secundaria a una taquicardia auricular focal e incesante tratada exitosamente con ivabradina, sin embargo el tratamiento médico no siempre es efectivo y seguro.

Dentro de las alternativas se encuentra la ablación con radiofrecuencia. Desde la introducción de la radiofrecuencia, el tratamiento de las taquiarritmias se ha llevado a cabo de manera segura y con resultados favorables. Son múltiples las taquiarritmias que se pueden tratar con esta técnica y en muchos casos, con mejoría hasta normalización de la función cardiaca posterior a la ablación con radiofrecuencia exitosa ${ }^{1,3}$.

En nuestro paciente se ocupó el sistema EnSite $\mathrm{Navx}^{\mathrm{TM}}$, herramienta que permite mapeo y navegación en 3 dimensiones de las arritmias. Esta tecnología facilita procedimientos de electrofisiología ya que provee identificación certera de la localización del foco arritmogénico, en este caso, de la localización del foco de taquicardia auricular y esclarecimiento del circuito de reentrada, incluso si la taquicardia auricular es no sostenida ${ }^{9,11}$.

La terapia con cirugía se pudiese considerar cuando la ablación con catéter no estuviese disponible como opción.

La recuperación de la función ventricular posterior al término o control de la arritmia puede ser completa, parcial e incluso ausente. Esto se relaciona al daño miocárdico presente en relación al tiempo de la taquiarritmia y a la cardiopatía estructural de base que difiere de paciente a paciente.

La mejoria de la función sistólica en modelos animales ocurre entre las 2-4 semanas del cese de la taquiarritmia. En estudios clínicos la recuperacion se observa al mes del término de la taquicardia, seguido por una mejoría más lenta que llega a su máximo a los 6-8 meses $^{2,4}$. En nuestro paciente se observó normalizacion de la funcion sistolica ventricular izquierda a los 6 meses posterior al 
procedimiento. Esta impresionante evolución del caso caracteriza la reversibilidad de las formas puras de taquicardiomiopatía.

En conclusión, el diagnóstico de taquicardiomiopatía puede ser difícil, debe ser sospechada cuando ocurre la asociación entre miocardiopatía dilatada idiopática y taquicardia crónica. Una vez que esta se sospecha, se debe intentar el cese de la arritmia ya que la disfunción ventricular izquierda puede revertir completamente al ser tratada en su fase inicial.

\section{Referencias}

1. Nakazato Y. Tachycardiomyopathy. Indian Pacing Electrophysiol J 2002; 2(4): 104-13

2. Fenelon G, Wijns W, Andries E, Brugada P. Tachycardiomyopathy: Mechanisms and Clinical Implications. Pacing Clin Electrophysiol 1996; 19 (1): 95-106.

3. Prabhu S, Dalvi B. Treatable Cardiomyopathies. Indian J Pediatr 2000; 67 (4): 279-282.

4. Umana E, Solares CA, Alpert MA. Tachycardia-induced cardiomyopathy. Am J Med 2003; 114 (1): 51-5.
5. Salemi VM, Arteaga E, Mady C. Recovery of systolic and diastolic function after ablation of incessant supraventricular tachycardia. Eur J Heart Fail 2005; 7 (7): 1177-9.

6. Phillips E, Levine S. Auricular fibrillation without other evidence of heart disease: A cause of reversible heart failure. Am J Med 1949; 7 (4): 478-89.

7. Walker NL, Cobbe SM, Birnie DH. Tachycardiomyopathy: a diagnosis not to be missed. Heart 2004; 90: e7

8. Bohora S, Lokhandwala Y, Parekh P, Vasavda A. Reversal of Tachycardiomyopathy Due to Left Atrial Tachycardia by Ivabradine. J Cardiovasc Electrophysiol 2010; 1-3.

9. Kettering K, Greil GF, Fenchel M, Kramer U, Weig HJ, Busch $\mathrm{M}$, et al. Catheter ablation of atrial fibrillation using the Navx-/Ensite-system and a CT-/MRI-guided approach. Clin Res Cardiol 2009; 98(5): 285-96.

10. Miyamoto K, Tsuchiya T, Narita S, Nagamoto Y, Yamaguchi T, Ando S, et al. Radiofrequency catheter ablation of ventricular tachyarrhythmia under navigation using EnSite array. Circ J 2010; 74 (7): 1322-31.

11. Eitel C, Hindricks G, Dagres N, Sommer P, Piorkowski C. EnSite Velocity cardiac mapping system: a new platform for 3D mapping of cardiac arrhythmias. Expert Rev Med Devices 2010; 7 (2): 185-92. 